\title{
Efficient Analysis of Photonic Crystal Slabs
}

\section{Mohammad Hasan Aram and Sina Khorasani ${ }^{\star}$}

School of Electrical Engineering, Sharif University of Technology, Tehran, Iran

\begin{abstract}
In this paper we propose an accelerated plane wave expansion method (PWEM) for calculation of slab photonic crystal (slab PC) modes. In this method instead of creating artificial periodicity in the direction of slab normal and solving the problem as a full 3D one, which is done in standard PWEM, we consider constant coefficients in 2D PWEM as functions of slab normal component. Then we eliminate electric and magnetic fields in Maxwell's equations in favor of electric and magnetic vector potentials to obtain equations that contain Laplace operator. Using the Green's function for Laplace operator we then change the problem from differential to integral form. Replacing the integral operators with their matrix representations and doing some matrix algebra we finally obtain a matrix which slab PC modes can be extracted from its eigenvalues. Advantages of this method over the common approach are its less computational complexity, faster creation of the eigenvalue problem, and faster convergence of eigenvalues as the matrix dimensions increase.
\end{abstract}

Keywords: Slab photonic crystal, Plane wave expansion method

Abbreviations: Slab PC: Slab Photonic Crystal; PWEM: Plane Wave Expansion Method; FDTD: Finite-difference Time-Domain; FEM: Finite-element method

\section{Introduction}

Photonic crystals (PCs) are optical nanostructures which their electric permittivity is periodic in one, two, or three dimensions. This periodic permittivity affects photons in the same manner as periodic potential affects electrons in ionic crystals. As a result, electromagnetic waves in these materials are in the form of Bloch waves. There are some finite and continuous frequency bands in which electromagnetic waves cannot propagate in PCs. Existence of these bands which are called photonic gaps, is the main property of PCs. Lord Rayleigh, the English physicist, observed photonic gaps in a one-dimensional PC for the first time in 1887 [1], but until a century later, when Yablono vitch predicted the existence of photonic gaps in three-dimensional PCs [2], there were no uses of them.

Today, PCs have found many applications in different fields, such as performance improvement of solar cells [3-6], omnidirectional surfaces [7], optical fibers and waveguides [8-12], optical cavities [1315], high efficient LEDs [16], and integrated optics [17,18].

As mentioned above there exists three types of photonic crystals based on their permittivity periodicity dimensions. Obviously, fabrication of ideally perfect two and three-dimensional PCs with photonic gaps in optical frequencies is impossible due to their infinite extensions. For the case of two-dimensional structures, however, the third dimension may be confined to a finite extent, which is referred to as the slab PC. The permittivity of a slab PC is still periodic in two-dimensions but unlike the two-dimensional PCs, it depends on the third component that is perpendicular to the slab surface. For all practical reasons, every two- or three-dimensional photonic structure must be ultimately fabricated in the form of slabs, calling for the need to a three-dimensional accurate, stable, and rapid numerical analysis.

There are many different methods to calculate PC modes theoretically. Some of them are time-domain like finite-difference time-domain (FDTD) and others are frequency-domain like finite-element method (FEM) and plane wave expansion (PWE). Unfortunately calculation of slab PC modes with any of these methods is computationally too demanding and complicated well beyond the capability of ordinary personal computers. Moreover, for attaining higher accuracy in these methods larger divisions and/or expansion terms are needed, which for three-dimensional structures and slabs result in huge matrices. Time-domain approaches such as FDTD suffers from inherent numerical dispersion and anisotropy which cannot be overcome by simple choice of smaller divisions. On the other hand, in the frequency-domain methods, calculation of eigenvalues for such matrices would be another challenge, and is normally unstable with the growth of matrix size.

This paper reports a novel scheme to calculate the eigen modes and band structure of photonic crystal slabs. Our proposed scheme is much more efficient and stable compared to the other widely used approaches, as demonstrated in the paper. Theoretical analysis of applications such as quantum cavity electrodynamics [19] of quantum dots embedded in photonic nanostructure highly rely on knowledge of mode frequencies, profiles, and density of states, all of which demand highly efficient mode extraction techniques for slabs.

\section{Accelerated and Stabilized PWEM}

Here we explain how we can calculate Bloch modes of slab PCs in a much faster and stable way. In this method the size of the final matrix which its eigenvalues should be calculated is not reduced necessarily, but its preparation is much faster than the standard approach. The point should be considered here is that the computation cost in slab PC problem is mostly related to matrix preparation rather that its eigenvalue calculation. In this method, we expand the fields in terms of vector and scalar potentials instead of electromagnetic observable quantities.

We begin with the relation,

$\mathbf{B}=\nabla \times \mathbf{A}$

*Corresponding author: Sina Khorasani, School of Electrical Engineering, Sharif University of Technology, Tehran, Iran, Tel: +98-21-6616-4352; E-mail: khorasani@sharif.edu

Received October 31, 2014; Accepted December 01, 2014; Published December 12,2014

Citation: Aram MH, Khorasani S (2014) Efficient Analysis of Photonic Crystal Slabs. J Laser Opt Photonics 1: 111. doi:10.4172/2469-410X.1000111

Copyright: () 2014 Aram MH, et al. This is an open-access article distributed under the terms of the Creative Commons Attribution License, which permits unrestricted use, distribution, and reproduction in any medium, provided the original author and source are credited. 
between magnetic flux density, $\mathbf{B}$ and magnetic vector potential, $\mathbf{A}$ According to Maxwell's equations we have

$$
\nabla \times \mathbf{B}=\mu\left(\mathbf{J}+\frac{\partial \mathbf{D}}{\partial t}\right)
$$

where $\mu$ is the space permeability, $\mathbf{J}$ is the electric current density, and D is the electric displacement field. If we take curl of both sides of (1) and use (2), we have

$$
\nabla \times \nabla \times \mathbf{A}=\mu\left(\mathbf{J}+\frac{\partial \mathbf{D}}{\partial t}\right)
$$

With the use of identity

$$
\nabla \times \nabla \times \mathbf{A} \equiv \nabla(\nabla \cdot \mathbf{A})-\nabla^{2} \mathbf{A}
$$

(3) can be written as

$$
\nabla(\nabla \cdot \mathbf{A})-\nabla^{2} \mathbf{A}=\mu\left(\mathbf{J}+\frac{\partial \mathbf{D}}{\partial t}\right)
$$

We assume Coulomb gauge, $\nabla \cdot \mathbf{A} \equiv 0$, for vector potential to simplify (5) as

$$
\nabla^{2} \mathbf{A}=-\mu\left(\mathbf{J}+\frac{\partial \mathbf{D}}{\partial t}\right)=-\mu \frac{\partial}{\partial t}(\varepsilon \mathbf{E}),
$$

where $\varepsilon$ is the electric permittivity. The second equation is written since there is no free electric current density in space. We also eliminated electric displacement in favor of electric field, E We can write electric field in its general form as

$$
\mathbf{E}=-\left(\nabla V+\frac{\partial \mathbf{A}}{\partial t}\right)
$$

Where $\mathbf{V}$ is the electric potential. Considering sinusoidal steady state, we can write (7) as

$$
\mathbf{E}=-(\nabla V+j \omega \mathbf{A})
$$

Combining (8) and (6) we have

$$
\nabla^{2} \mathbf{A}=\frac{\varepsilon_{r}}{c^{2}} \frac{\partial}{\partial t}(\nabla V+j \omega \mathbf{A})=\frac{\varepsilon_{r}}{c^{2}}\left(j \omega \nabla V-\omega^{2} \mathbf{A}\right)
$$

According to Gauss's law, when there is no free electric charge in space, $\rho=0$, electric field satisfies

$$
\nabla \cdot(\varepsilon \mathbf{E})=\nabla \varepsilon \cdot \mathbf{E}+\varepsilon \nabla \cdot \mathbf{E}=0
$$
obtain

Replacing $\mathbf{E}$ with its value from (8) and using Coulomb gauge we

$$
\nabla \varepsilon \cdot(\nabla V+j \omega \mathbf{A})+\varepsilon \nabla \cdot(\nabla V+j \omega \mathbf{A})=\nabla \varepsilon \cdot(\nabla V+j \omega \mathbf{A})+\varepsilon \nabla^{2} V=0
$$

We can write (11) in a different form as

$$
\nabla^{2} V=-\frac{\nabla \varepsilon_{r}}{\varepsilon_{r}} \cdot(\nabla V+j \omega \mathbf{A})=-\nabla \ln \left(\varepsilon_{r}\right) \cdot(\nabla V+j \omega \mathbf{A})
$$

An example of a slab PC that we want to calculate its modes is shown in Figure 1. As can be seen, permittivity of space and its logarithm can be written as Fourier series with coefficients depend on $\mathrm{z}$ component,

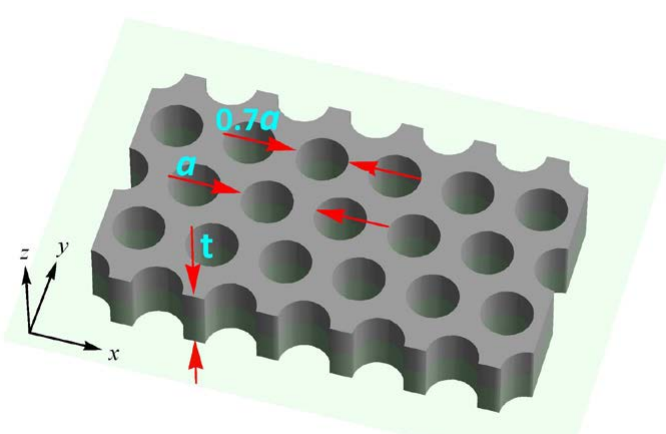

Figure 1: Slab PC which its modes is calculated in this section. This crystal is formed by carving air cylinders inside a Silicon substrate with diameters equal 0.7 lattice constant. Slab thickness is $0.6 a$.

$$
\begin{aligned}
& \varepsilon_{r}(\vec{r})=\sum_{\vec{G}} \varepsilon_{r_{\vec{G}}}(z) e^{-j \vec{G} \cdot \vec{r}_{x y}} \\
& \ln \left(\varepsilon_{r}(\vec{r})\right)=s(z) \sum_{\vec{G}} \chi_{\vec{G}} e^{-j \vec{G} \cdot \vec{r}_{x y}}
\end{aligned}
$$

Here $\vec{G}=m \vec{g}_{1}+n \vec{g}_{2}, \quad \vec{g}_{i}(i=1,2)$ are primitive reciprocal lattice vectors of a two-dimensional crystal with the same permittivity pattern, where $\mathrm{m}, \mathrm{n}$ are integers and $\varepsilon_{r_{\vec{G}}}(z)$ and $s(z)$ are defined as

$$
\begin{aligned}
& \varepsilon_{r_{\vec{G}}}(z)= \begin{cases}\varepsilon_{r_{\vec{G}}}(0) & \text { if } \quad|z| \leq t / 2 \\
1 & \text { if } \quad|z|>t / 2, \vec{G}=0 \\
0 & \text { if } \quad|z|>t / 2, \vec{G} \neq 0\end{cases} \\
& s(z)=\left\{\begin{array}{lll}
1 & \text { if } & |z| \leq t / 2 \\
0 & \text { if } & |z|>t / 2
\end{array}\right.
\end{aligned}
$$

According to Bloch theorem, magnetic vector potential and electric potential in this problem are of the form

$$
\begin{aligned}
\mathbf{A}(\vec{r}) & =e^{-j \vec{k} \cdot \vec{r}_{x y}} \sum_{\vec{G}} \mathbf{A}_{\vec{G}}(z) e^{-j \vec{G} \cdot \vec{r}_{x y}} \\
V(\vec{r}) & =e^{-j \vec{k} \cdot \vec{r}_{x y}} \sum_{\vec{G}} V_{\vec{G}}(z) e^{-j \vec{G} \cdot \vec{r}_{x y}}
\end{aligned}
$$

where $\vec{\kappa}$ is the Bloch wave vector that is supposed to be in the plane of slab, i.e. $\vec{\kappa}_{z}=0$. Replacing $\mathbf{A}(\vec{r}), V(\vec{r})$, and $\varepsilon_{r}(\vec{r})$ in (9) with their values from (15) and (13) and assuming, $c=1$, according to the conventional normalized angular frequency scheme, we obtain

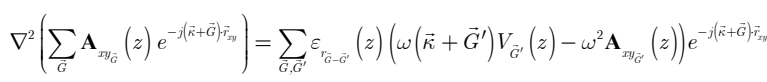

for the in-plane, $x y$, component of vector potential. We can change (16) from differential to integral form, by using Laplace operator Green's function [20],

$$
\begin{aligned}
& G\left(\vec{r}, \vec{r}^{\prime}\right)=\frac{-1}{4 \pi\left|\vec{r}-\vec{r}^{\prime}\right|} \\
& \sum_{\vec{G}} \mathbf{A}_{x y_{\vec{G}}}(z) e^{-j(\vec{k}+\vec{G}) \vec{r}_{x y}}=\int_{-\infty}^{\infty} \int_{S^{\prime}} \sum_{\vec{G}, \vec{G}^{\prime}} \varepsilon_{r_{\vec{G}-\overrightarrow{G^{\prime}}}}\left(z^{\prime}\right)\left(\omega^{2} \mathbf{A}_{x y_{\overrightarrow{U^{\prime}}}}\left(z^{\prime}\right)-\omega\left(\vec{\kappa}+\vec{G}^{\prime}\right) V_{\vec{G}^{\prime}}\left(z^{\prime}\right)\right) \\
& \cdot \frac{e^{-j(\vec{r}+\vec{G}) \vec{r}_{x y}}}{4 \pi \sqrt{\left|\vec{r}_{x y}-\vec{r}_{x y}^{\prime}\right|^{2}+\left(z-z^{\prime}\right)^{2}}} d^{2} r_{x y}^{\prime} d z^{\prime}
\end{aligned}
$$


With the help of identity [21]

$$
\int \frac{e^{-j \vec{K} \cdot \vec{r}}}{\sqrt{|\vec{r}|^{2}+z^{2}}} d^{2} \vec{r} \equiv \frac{2 \pi}{|\vec{K}|} e^{-|\vec{K}| z \mid}
$$

(18) simplifies to

$$
\begin{aligned}
& \sum_{\vec{G}} \mathbf{A}_{x y_{\vec{G}}}(z) e^{-j(\vec{k} \cdot \vec{G}) \cdot \vec{r}_{x y}}=\sum_{\vec{G}, G^{\prime}} \int_{-\infty}^{\infty} \varepsilon_{r_{\hat{G}-\vec{G}^{\prime}}}\left(z^{\prime}\right)\left(\omega^{2} \mathbf{A}_{x y_{G^{\prime}}}\left(z^{\prime}\right)-\omega\left(\vec{\kappa}+\vec{G}^{\prime}\right) V_{\vec{G}^{\prime}}\left(z^{\prime}\right)\right) \\
& \frac{e^{-|\vec{k}+\vec{G}| z-z \mid z^{-j}} e^{-j(\vec{k}+\vec{G}) \cdot \overrightarrow{x_{y}}}}{2|\vec{\kappa}+\vec{G}|} d z^{\prime}
\end{aligned}
$$

Again because of orthogonality of $e^{-j \vec{G} \cdot \vec{r}}$ for all $\vec{G}$ in reciprocal lattice, we can further simplify (20) as

$$
\mathbf{A}_{x y_{\hat{G}^{\prime}}}(z)=\sum_{\vec{G}^{\prime}} \int_{-\infty}^{\infty} \frac{\varepsilon_{r_{\vec{g}-\vec{G}^{\prime}}}\left(z^{\prime}\right)\left(\omega^{2} \mathbf{A}_{x y_{\hat{G}^{\prime}}}\left(z^{\prime}\right)-\omega\left(\vec{\kappa}+\vec{G}^{\prime}\right) V_{\vec{G}^{\prime}}\left(z^{\prime}\right)\right)}{2|\vec{\kappa}+\vec{G}|} e^{-|\vec{k}+\vec{G}| z-z^{\prime} \mid} d z^{\prime}
$$

If we go the same process for the $z$ component of (9) we obtain

$$
\mathbf{A}_{z_{\tilde{G}}}(z)=\sum_{\vec{G}^{\prime}} \int_{-\infty}^{\infty} \frac{\varepsilon_{r_{\vec{G}-\vec{G}^{\prime}}}\left(z^{\prime}\right)\left(\omega^{2} \mathbf{A}_{z_{\vec{\sigma}^{\prime}}}\left(z^{\prime}\right)-j \omega V_{\vec{G}^{\prime}}^{\prime}\left(z^{\prime}\right)\right)}{2|\vec{\kappa}+\vec{G}|} e^{-|\vec{\kappa}+\vec{G}| z-z^{\prime} \mid} d z^{\prime}
$$

where $V_{\vec{G}^{\prime}}^{\prime}\left(z^{\prime}\right)=\partial V_{\vec{G}^{\prime}}\left(z^{\prime}\right) / \partial z^{\prime}$. The last equation we need to be able to solve our slab PC problem is obtained by replacing $\mathbf{A}(\vec{r}), V(\vec{r})$, and $\ln \left(\varepsilon_{r}(\vec{r})\right)$ in (12) with their values from (15) and (13). Again by using Laplace operator Green's function and doing some simplification we reach

$$
\begin{array}{r}
V_{\vec{G}}(z)=\sum_{\vec{G}^{\prime}} x_{\vec{G}-\vec{G}} \int_{-\infty}^{\infty}\left[\frac{s\left(z^{\prime}\right)\left(\vec{G}-\vec{G}^{\prime}\right) \cdot\left(-\left(\vec{\kappa}+\vec{G}^{\prime}\right) V_{\vec{G}^{\prime}}\left(z^{\prime}\right)+\omega \mathbf{A}_{x y_{\vec{G}^{\prime}}}\left(z^{\prime}\right)\right)}{2|\vec{\kappa}+\vec{G}|}\right. \\
\left.+\frac{s^{\prime}\left(z^{\prime}\right)\left(V_{\vec{G}^{\prime}}^{\prime}\left(z^{\prime}\right)+j \omega A_{z_{\overrightarrow{\sigma^{\prime}}}}\left(z^{\prime}\right)\right)}{2|\vec{\kappa}+\vec{G}|}\right] e^{-|\vec{\kappa}+\vec{G}| z-z^{\prime} \mid} d z^{\prime}
\end{array}
$$

We now take dot product of both sides of (21) by the unit vector $(\vec{\kappa}+\vec{G})^{\perp} /|\vec{\kappa}+\vec{G}|$ to obtain

$$
\begin{aligned}
& A_{\perp_{\vec{G}}}(z)=\omega^{2} \sum_{\vec{G}^{\prime}} \int_{-\infty}^{\infty} \frac{\varepsilon_{r_{\vec{G}-\vec{G}^{\prime}}}\left(z^{\prime}\right)\left(\vec{\kappa}+\vec{G}^{\prime}\right)^{\perp} \cdot(\vec{\kappa}+\vec{G})^{\perp}}{2|\vec{\kappa}+\vec{G}|^{2}\left|\vec{\kappa}+\vec{G}^{\prime}\right|} A_{\perp_{\overrightarrow{\sigma^{\prime}}}}\left(z^{\prime}\right) e^{-|\vec{k}+\vec{G}| z-z^{\prime} \mid} d z^{\prime} \\
& +\omega^{2} \sum_{\vec{G}^{\prime}} \int_{-\infty}^{\infty} \frac{\varepsilon_{r_{G-\sigma^{\prime}}}\left(z^{\prime}\right)\left(\vec{\kappa}+\vec{G}^{\prime}\right) \cdot(\vec{\kappa}+\vec{G})^{\perp}}{2|\vec{\kappa}+\vec{G}|^{2}\left|\vec{\kappa}+\vec{G}^{\prime}\right|} A_{k^{\prime}}\left(z^{\prime}\right) e^{-|\vec{\kappa}+\vec{G}| z-z^{\prime \prime} \mid} d z^{\prime} \\
& -\omega \sum_{\vec{G}^{\prime}} \int_{-\infty}^{\infty} \frac{\varepsilon_{r_{\vec{c}-\vec{\sigma}^{\prime}}}\left(z^{\prime}\right)\left(\vec{\kappa}+\overrightarrow{G^{\prime}}\right) \cdot(\vec{\kappa}+\vec{G})^{\perp}}{2|\vec{\kappa}+\vec{G}|^{2}} V_{\vec{G}^{\prime}}\left(z^{\prime}\right) e^{-|\vec{k}+\vec{G}| z-z^{\prime} \mid} d z^{\prime}
\end{aligned}
$$

where $(\vec{\kappa}+\vec{G})^{\perp}$ is perpendicular to $(\vec{\kappa}+\vec{G})$ but with the same magnitude. $A_{l_{\vec{G}}}(z)$ and $A_{\perp_{\vec{G}}}(z)$ are projections of $\mathbf{A}_{x y_{\vec{G}}}(z)$ onto the direction of wave propagation, $(\vec{\kappa}+\vec{G})$, and direction of perpendicular to wave propagation and slab normal respectively. We can write (24) in matrix form

$$
\left|A_{\perp_{\bar{G}}}(z)\right\rangle=\omega^{2}\left([\mathbb{K} 1]\left|A_{\perp_{\bar{G}^{\prime}}}\left(z^{\prime}\right)\right\rangle+[\mathbb{K} 2]\left|A_{\|_{\bar{G}^{\prime}}}\left(z^{\prime}\right)\right\rangle\right)-\omega[\mathbb{K} 3]\left|V_{\vec{G}^{\prime}}\left(z^{\prime}\right)\right\rangle
$$

where elements of matrices $[\mathbb{K} 1],[\mathbb{K} 2]$, and $[\mathbb{K} 3]$ are integral operators as follows,

$$
\begin{aligned}
& {[\mathbb{K} 1]_{\vec{G}_{i}, \vec{G}_{j}}\left(z, z^{\prime}\right)=\int_{-\infty}^{\infty} \frac{\varepsilon_{r_{\hat{G}_{i}-\vec{G}_{j}}}\left(z^{\prime}\right)\left(\vec{\kappa}+\vec{G}_{j}\right)^{\perp} \cdot\left(\vec{\kappa}+\vec{G}_{i}\right)^{\perp}}{2\left|\vec{\kappa}+\vec{G}_{i}\right|^{2}\left|\vec{\kappa}+\vec{G}_{j}\right|} e^{-\left|\vec{\kappa}+\vec{G}_{i}\right|\left|z-z^{\prime}\right|} d z^{\prime},} \\
& {[\mathbb{K} 2]_{\vec{G}_{i}, \vec{G}_{j}}\left(z, z^{\prime}\right)=\int_{-\infty}^{\infty} \frac{\varepsilon_{r_{\hat{G}_{i}-\vec{G}_{j}}}\left(z^{\prime}\right)\left(\vec{\kappa}+\vec{G}_{j}\right) \cdot\left(\vec{\kappa}+\vec{G}_{i}\right)^{\perp}}{2\left|\vec{\kappa}+\vec{G}_{i}\right|^{2}\left|\vec{\kappa}+\vec{G}_{j}\right|} e^{-\left|\vec{\kappa}+\vec{G}_{i}\right| z-z^{\prime} \mid} d z^{\prime},} \\
& {[\mathbb{K} 3]_{\vec{G}_{i}, \vec{G}_{j}}\left(z, z^{\prime}\right)=\int_{-\infty}^{\infty} \frac{\varepsilon_{r_{\vec{G}_{i}-\vec{G}_{j}}}\left(z^{\prime}\right)\left(\vec{\kappa}+\vec{G}_{j}\right) \cdot\left(\vec{\kappa}+\vec{G}_{i}\right)^{\perp}}{2\left|\vec{\kappa}+\vec{G}_{i}\right|^{2}} e^{-\left|\vec{\kappa}+\vec{G}_{i}\right|\left|z-z^{\prime}\right|} d z^{\prime}}
\end{aligned}
$$

We can now take dot product of both sides of (21) by the unit vector $(\vec{\kappa}+\vec{G}) /|\vec{\kappa}+\vec{G}|$ to obtain

$$
\left|A_{l_{\tilde{G}}}(z)\right\rangle=\omega^{2}\left([\mathbb{L} 1]\left|A_{\perp_{\vec{\sigma}^{\prime}}}\left(z^{\prime}\right)\right\rangle+[\mathbb{L} 2]\left|A_{{\overrightarrow{\sigma^{\prime}}}^{\prime}}\left(z^{\prime}\right)\right\rangle\right)-\omega[\mathbb{L} 3]\left|V_{\vec{G}^{\prime}}\left(z^{\prime}\right)\right\rangle(27)
$$

where elements of matrices $[\mathbb{L} 1],[\mathbb{L} 2]$, and $[\mathbb{L} 3]$ are integral operators

$$
\begin{aligned}
& {[\mathbb{L} 1]_{\vec{G}_{i}, \vec{G}_{j}}\left(z, z^{\prime}\right)=\int_{-\infty}^{\infty} \frac{\varepsilon_{r_{\vec{G}_{i}-\vec{G}_{j}}}\left(z^{\prime}\right)\left(\vec{\kappa}+\vec{G}_{j}\right)^{\perp} \cdot\left(\vec{\kappa}+\vec{G}_{i}\right)}{2\left|\vec{\kappa}+\vec{G}_{i}\right|^{2}\left|\vec{\kappa}+\vec{G}_{j}\right|} e^{-\left|\vec{\kappa}+\vec{G}_{i}\right|\left|z-z^{\prime}\right|} d z^{\prime},} \\
& {[\mathbb{L} 2]_{\vec{G}_{i}, \vec{G}_{j}}\left(z, z^{\prime}\right)=\int_{-\infty}^{\infty} \frac{\varepsilon_{r_{\vec{G}_{i}-\vec{G}_{j}}}\left(z^{\prime}\right)\left(\vec{\kappa}+\vec{G}_{j}\right) \cdot\left(\vec{\kappa}+\vec{G}_{i}\right)}{2|\vec{\kappa}+\vec{G}|^{2}|\vec{\kappa}+\vec{G}|} e^{-\left|\vec{\kappa}+\vec{G}_{i}\right|\left|z-z^{\prime}\right|} d z^{\prime},} \\
& {[\mathbb{L} 3]_{\vec{G}_{i}, \vec{G}_{j}}\left(z, z^{\prime}\right)=\int_{-\infty}^{\infty} \frac{\varepsilon_{\vec{G}_{\vec{G}_{i}-\vec{G}_{j}}}\left(z^{\prime}\right)\left(\vec{\kappa}+\vec{G}_{j}\right) \cdot\left(\vec{\kappa}+\vec{G}_{i}\right)}{2\left|\vec{\kappa}+\vec{G}_{i}\right|^{2}} e^{-\left|\vec{\kappa}+\overrightarrow{G_{i}}\right|\left|z-z^{\prime}\right|} d z^{\prime}}
\end{aligned}
$$

We can also write (23) in matrix form

$$
\begin{aligned}
& \left|V_{\widetilde{G}}(z)\right\rangle=-[\mathbb{M} 1]\left|V_{\tilde{G}^{\prime}}\left(z^{\prime}\right)\right\rangle+[\mathbb{M} 2]\left|V_{\tilde{G}^{\prime}}^{\prime}\left(z^{\prime}\right)\right\rangle+\omega[\mathbb{M} 3]\left|A_{\lrcorner_{\mathscr{G}^{\prime}}}\left(z^{\prime}\right)\right\rangle+\omega[\mathbb{M} 4]\left|A_{k^{\prime}}\left(z^{\prime}\right)\right\rangle \\
& +j \omega[\mathbb{M} 2]\left|A_{z_{g^{\prime}}}\left(z^{\prime}\right)\right\rangle
\end{aligned}
$$

where elements of matrices $[\mathbb{M} 1],[\mathbb{M} 2],[\mathbb{M} 3]$, and $[\mathbb{M} 4]$ are integral operators

$$
\begin{aligned}
& {[\mathbb{M} 1]_{\vec{G}_{i}, \vec{G}_{j}}\left(z, z^{\prime}\right)=\int_{-t / 2}^{t / 2} \frac{\chi_{\vec{G}_{i}-\vec{G}_{j}}\left(\vec{\kappa}+\vec{G}_{j}\right) \cdot\left(\vec{G}_{i}-\vec{G}_{j}\right)}{2\left|\vec{\kappa}+\vec{G}_{i}\right|} e^{-\left|\vec{\kappa}+\vec{G}_{i}\right| z-z^{\prime} \mid} d z^{\prime},} \\
& {[\mathbb{M} 2]_{\vec{G}_{i}, \vec{G}_{j}}\left(z, z^{\prime}\right)=\int_{-t / 2}^{t / 2} \frac{s^{\prime}\left(z^{\prime}\right) \chi_{\vec{G}_{i}-\vec{G}_{j}} e^{-\left|\vec{\kappa}+\vec{G}_{i}\right| z-z^{\prime} \mid} d z^{\prime},}{2\left|\vec{\kappa}+\vec{G}_{i}\right|}} \\
& {[\mathbb{M} 3]_{\vec{G}_{i}, \vec{G}_{j}}\left(z, z^{\prime}\right)=\int_{-t / 2}^{t / 2} \frac{\chi_{\vec{G}_{i}-\vec{G}_{j}}\left(\vec{\kappa}+\vec{G}_{j}\right)^{\perp} \cdot\left(\vec{G}_{i}-\vec{G}_{j}\right)}{2\left|\vec{\kappa}+\vec{G}_{i}\right|\left|\vec{\kappa}+\vec{G}_{j}\right|} e^{-\left|\vec{k}+\vec{G}_{i}\right| \vec{z}^{\prime}-z^{\prime} \mid} d z^{\prime},} \\
& {[\mathbb{M} 4]_{\vec{G}_{i}, \vec{G}_{j}}\left(z, z^{\prime}\right)=\int_{-t / 2}^{t / 2} \frac{\chi_{\vec{G}_{i}-\vec{G}_{j}}\left(\vec{\kappa}+\vec{G}_{j}\right) \cdot\left(\vec{G}_{i}-\vec{G}_{j}\right)}{2\left|\vec{\kappa}+\vec{G}_{i}\right|\left|\vec{\kappa}+\vec{G}_{j}\right|} e^{-\left|\vec{k}+\vec{G}_{i}\right|\left|z-z^{\prime}\right|} d z^{\prime}}
\end{aligned}
$$

If we can expand even and odd functions of $z$ component in our equations respectively by cosine or sine series within a distance around the slab, then we can easily write a linear equation which relates derivative of a function by itself. For instance we can write

$$
\left|V_{\vec{G}}^{\prime}(z)\right\rangle=[N]\left|V_{\vec{G}}(z)\right\rangle
$$

where $[N]$ is a block diagonal matrix like

$$
[N]=\left(\begin{array}{cccc}
{[F]} & 0 & 0 & \ldots \\
0 & {[F]} & 0 & \ldots \\
0 & 0 & {[F]} & \ldots \\
\vdots & \vdots & \vdots & \ddots
\end{array}\right)
$$


where $[F]$ equals

$[F]=\left(\begin{array}{ccccc}0 & \frac{-2 \pi}{l} & 0 & 0 & \ldots \\ 0 & 0 & 2 \times \frac{-2 \pi}{l} & 0 & \ldots \\ 0 & 0 & 0 & 3 \times \frac{-2 \pi}{l} & \ldots \\ \vdots & \vdots & \vdots & \vdots & \ddots\end{array}\right)$

and 1 is the distance around the slab which we have written the cosine and sine series in it. Combining (29) and (31) we can write $\left|V_{\vec{G}}(z)\right\rangle$ as a function of $\left|\mathbf{A}_{\vec{G}}(z)\right\rangle$,

$$
\left|V_{\overparen{G}}(z)\right\rangle=\omega[J]^{-1}\left([\mathbb{M} 3]\left|A_{\perp_{\widehat{G}^{\prime}}}\left(z^{\prime}\right)\right\rangle+[\mathbb{M} 4]\left|A_{l_{\bar{c}^{\prime}}}\left(z^{\prime}\right)\right\rangle+j[\mathbb{M} 2]\left|A_{z_{\vec{G}^{\prime}}}\left(z^{\prime}\right)\right\rangle\right)
$$

Where $[\mathbb{J}]=([I]+[\mathbb{M} 1]-[\mathbb{M} 2][N])$. Replacing $\left|V_{\vec{G}}(z)\right\rangle$ in (25) and (27) by its value from (34) we obtain

$$
\begin{aligned}
& \left|A_{\perp_{\grave{G}}}(z)\right\rangle=\omega^{2}\left([\mathbb{O} 1]\left|A_{\perp_{\bar{G}}}\left(z^{\prime}\right)\right\rangle+[\mathbb{O} 2]\left|A_{l_{\bar{c}}}\left(z^{\prime}\right)\right\rangle-j[\mathbb{O} 3]\left|A_{z_{\bar{G}}}\left(z^{\prime}\right)\right\rangle\right), \\
& \left|A_{b_{b}}(z)\right\rangle=\omega^{2}\left([\mathbb{P} 1]\left|A_{\perp_{\tilde{G}}}\left(z^{\prime}\right)\right\rangle+[\mathbb{P} 2]\left|A_{\downarrow_{b}}\left(z^{\prime}\right)\right\rangle-j[\mathbb{P} 3]\left|A_{z_{\tilde{G}}}\left(z^{\prime}\right)\right\rangle\right)
\end{aligned}
$$

where

$$
\begin{aligned}
& {[\mathbb{O} 1]=[\mathbb{K} 1]-[\mathbb{K} 3][\mathbb{J}]^{-1}[\mathbb{M} 3]} \\
& {[\mathbb{O} 2]=[\mathbb{K} 2]-[\mathbb{K} 3][\mathbb{J}]^{-1}[\mathbb{M} 4]} \\
& {[\mathbb{O} 3]=[\mathbb{K} 3][\mathbb{J}]^{-1}[\mathbb{M} 2]} \\
& {[\mathbb{P} 1]=[\mathbb{L} 1]-[\mathbb{L} 3][\mathbb{J}]^{-1}[\mathbb{M} 3]} \\
& {[\mathbb{P} 2]=[\mathbb{L} 2]-[\mathbb{L} 3][\mathbb{J}]^{-1}[\mathbb{M} 4]} \\
& {[\mathbb{P} 3]=[\mathbb{L} 3][\mathbb{J}]^{-1}[\mathbb{M} 2]}
\end{aligned}
$$

Again we use Coulomb gauge to eliminate one of $\left|\mathbf{A}_{\vec{G}}(z)\right\rangle$ components in favor of the others. According to this gauge we have

$$
\begin{aligned}
\nabla \cdot \mathbf{A}=\nabla \cdot\left(\sum_{\vec{G}} \mathbf{A}_{\vec{G}}(z) e^{-j(\vec{k}+\vec{G}) \cdot r_{x y}}\right)=\nabla_{x y} \cdot\left(\sum_{\vec{G}} \mathbf{A}_{x y_{\hat{G}}}(z) e^{-j(\vec{k}+\vec{G}) \cdot \vec{r}_{x y}}\right) \\
+\sum_{\vec{G}} A_{z_{\vec{G}}^{\prime}}^{\prime}(z) e^{-j(\vec{k}+\vec{G}) \cdot \vec{r}_{x y}} \equiv 0
\end{aligned}
$$

Therefore we conclude

$\forall \vec{G}: \quad A_{z_{\vec{G}}}^{\prime}(z)=j|\vec{\kappa}+\vec{G}| A_{\left.\right|_{\vec{G}}}(z)$

We can write (38) in matrix form as

$$
[Q]\left|A_{z_{\tilde{G}}}(z)\right\rangle=j[R]\left|A_{l_{\vec{G}}}(z)\right\rangle
$$

provided that $A_{z_{\overparen{G}}}(z)$ can be approximated by cosine or sine series. Combining (35) and (39) we finally obtain the eigenvalue equation for the components of magnetic vector potential as

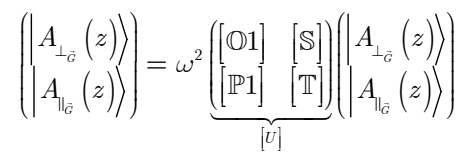

where

$$
[\mathbb{S}]=[\mathbb{O} 2]+[\mathbb{O} 3][\mathbb{Q}]^{-1}[\mathbb{R}] \quad, \quad[\mathbb{T}]=[\mathbb{P} 2]+[\mathbb{P} 3][\mathbb{Q}]^{-1}[\mathbb{R}]
$$

We can calculate the normalized angular frequency $\omega$ as a function of Bloch wave vector, $\vec{\kappa}$, using the equation

$$
\omega(\vec{\kappa})=\sqrt{\frac{1}{\text { Eigenvalue }\{[U(\vec{\kappa})]\}}}
$$

Using (42) we have calculated TE-like band structure of the slab PC shown in Figure 1 with $t=0.6 a$. This band structure is shown in Figure 2 with Blue dots. For comparison result of FDTD method is also plotted with red circles.

\section{Performance Comparison}

As mentioned in the above, the main advantage of our method over the common one is that its results converge faster as the size of the final matrix increase and this matrix is also prepared much more efficiently. Counter intuitively, calculation of the matrix operators in (40) and setup of the eigenvalue problem is much faster compared to the standard approach. The reason is as follows. Suppose that one would utilize $\mathbf{N}$ expansion terms along each direction. Then, the size of final eigenvalue problem in both standard and our new method would be identically $N^{3}$. While the standard PWEM would require $N^{3}$ individual calculation

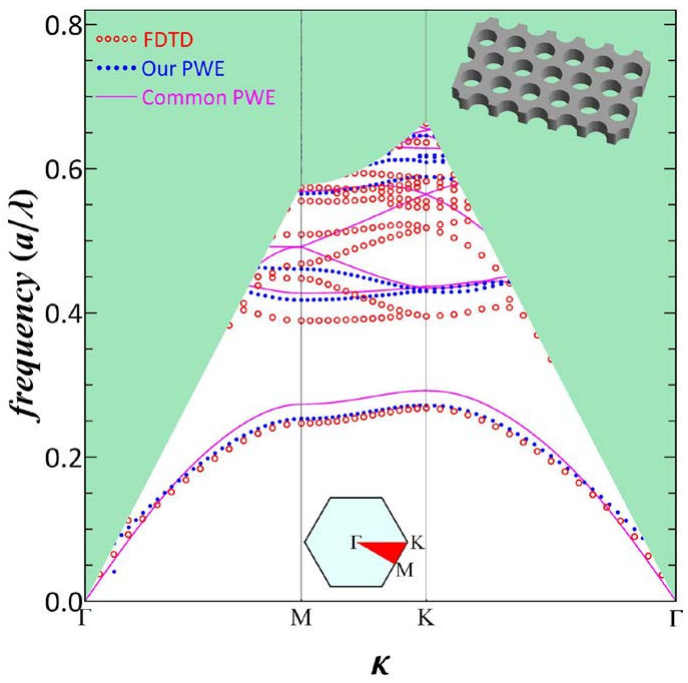

Figure 2: TE-like band structure of the slab PC shown in Figure.1 with $t=0.6 a$. Blue dots are calculated from (42), red circles are results of FDTD, and magenta curves are obtained from three-dimensional standard PWEM.

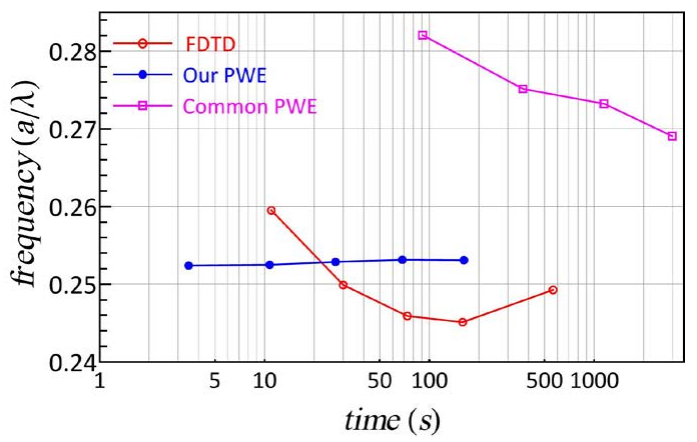

Figure 3: Comparison of our method with other ones. Frequency of the first mode of slab PC of previous section at $\mathrm{M}^{(1)}$ in reciprocal lattice against the time it took to calculate it. Red line and circles are related to FDTD, blue line and dots are results of our method, and common method is shown with magenta line and squares. 
subroutines to setup the full coefficients matrix, our proposed scheme would require only $N^{2}$ calculations given for a sub-matrix which apart from a scalar multiplication repeats to complete the full-sized matrix having the size $N^{3}$. Put in other words, while the standard PWEM is an algorithm with computational complexity $O\left(N^{3}\right)$, this proposed algorithm has a computational complexity $N^{2}$. It is exactly for this reason that in practice our scheme turns out to be much more efficient and stable compared to the standard PWEM. To illustrate this we have plotted the first mode frequency of the slab PC of previous section at the high-symmetry point $\mathrm{M}^{(1)}$ against the time it took to calculate it using our method, the standard PWEM, and FDTD in Figure 3. As it can be seen our method converges to the final result much faster within few seconds while for the FDTD and standard PWEM, one would need roughly at least half an hour to obtain a trustable result.

\section{Conclusion}

In this paper we introduced a new method to find modes of a slab PC. In this method which is based on PWE, unlike the standard PWEM that change the slab PC structure to obtain a three-dimensional crystal, we consider the slab as a two-dimensional crystal but with Fourier series coefficients depend on slab normal component. We showed that with this method we can calculate slab PCs modes much faster and no super-computing is needed for it.

\section{References}

1. Joannopoulos JD, Johnson SG, Winn JN, Meade RD (2008) Photonic Crystals: Molding the Flow of Light. (2ndedn), Princeton University Press: Princeton.

2. Yablonovitch E (1987) Inhibited Spontaneous Emission in Solid-State Physics and Electronics. Phys Rev Lett 58: 2059-2062.

3. Zhou D, Biswas R (2008) Photonic crystal enhanced light-trapping in thin film solar cells. J Appl Phys 103.

4. Mutitu JG, Shi S, Chen C, Creazzo T, Barnett A, et al. (2008) Thin film silicon solar cell design based on photonic crystal and diffractive grating structures. Optics Express 16: 15238-15248.

5. Bermel P, Luo C, Zeng L, Kimerling LC, Joannopoulos JD (2007) Improving thin-film crystalline silicon solar cell efficiencies with photonic crystals. Optics Express 15: 16986-17000.
6. Zeng L, Bermel P, Yi Y, Alamariu BA, Broderick KA, et al. (2008) Demonstration of enhanced absorption in thin film Si solar cells with textured photonic crystal back reflector. Appl Phys Lett 93: 221105.

7. Winn JN, Fink Y, Fan S, Joannopoulos JD (1998) Omnidirectional reflection from a one-dimensional photonic crystal. Optics Letters 23: 1573-1575.

8. Cerqueira SA Jr (2010) Recent progress and novel applications of photonic crystal fibers. Rep Prog Phys 73: 024401.

9. Johnson SG, Villeneuve PR, Fan S, Joannopoulos JD (2000) Linear waveguides in photonic-crystal slabs. Phys Rev B 62 : 8212- 8222.

10. Lin S, Chow E, Hietala V, Villeneuve PR, Joannopoulos JD (1998) Experimental Demonstration of Guiding and Bending of Electromagnetic Waves in a Photonic Crystal. Science 282: 274-276.

11. Loncar M, Doll T, Vuckovic J, Scherer A (2000) Design and Fabrication of Silicon Photonic Crystal Optical Waveguides. J Lightw Technol 8: 1402-1411.

12. Chutinan A, Noda S (1999) Highly confined waveguides and waveguide bends in three-dimensional photonic crystal. Appl Phys Lett 75: 3739-3741.

13. Altug H , Englund D, Vuckovic J (2006) Ultrafast photonic crystal nanocavity laser. Nat Phys 2: 484-488.

14. Hirayama $H$, Hamano $T$, Aoyagi $Y(1996)$ Novel surface emitting laser diode using photonic band-gap crystal cavity. Appl Phys Lett 69: 791-793.

15. Akahane Y, Asano T, Song B, Noda S (2003) High-Q photonic nanocavity in a two-dimensional photonic crystal. Nature 425: 944-947.

16. Ichikawa H, Baba T (2004) Efficiency enhancement in a lightemitting diode with a two-dimensional surface grating photonic crystal. Appl Phys Lett 84: pp. 457-459.

17. McNab SJ, Moll N, Vlasov YA (2003) Ultra-low loss photonic integrated circuit with membrane-type photonic crystal waveguides. Optics Express 11: 2927 2939.

18. Soljačić M, Luo C, Joannopoulos JD (2003) Nonlinear photonic crystal micro devices for optical integration. Optics Letters 28: 637-639.

19. Shahraki MA, Khorasani S, Aram MH (2013) Simulation of Multipartite Cavity Quantum Electro Dynamics. IEEE Journal of Quantum Electronics 49: 10661079.

20. Bayin S (2006) Mathematical Methods in Science and Engineering. (1stedn), John Wiley and Sons, New Jersey.

21. Zandi H, Hosseini A, Khorasani S, Mehrany K, Rashidian B, et al. (2008) Plasmonic Propagation Modes of a Structured Two dimensional Conducting Interface. Journal of Optics A: Pure and Applied Optics 10: 025202. 